\title{
Keys to drug sensitivity from updated functional work flows
}

\author{
Stephen E. Kurtz ${ }^{1}$ and Jeffrey W. Tyner ${ }^{1,2}$
}

${ }^{1}$ Division of Hematology and Medical Oncology and 'Department of Cell, Developmental and Cancer Biology, Knight Cancer Institute, Oregon Health and Science University, Portland, OR, USA

E-mail: JEFFREYW. TYNER - tynerj@ohsu.edu

doi:10.3324/haematol.2020.250662

A primary goal of cancer therapy is to match patients with the most appropriate drug regimens. Identifying characteristics of patients who respond to therapies and devising alternative strategies for non-responsive patients are important clinical considerations. Next generation sequencing (NGS) has provided a central technology to reveal genetic alterations and guide this process. Additionally, the development of cancer therapies that target specific signaling pathways and subcellular components has increased the opportunity for matching patients with molecularly targeted drugs. In practice, however, limitations in understanding the relationship between cancer genotypes and their corresponding phenotypes have hindered this process; somatic cancer mutations do not always reliably suggest therapies. Indeed, in some cases, targeted drugs have shown clinical utility when matched to cell phenotypes rather than somatic genotype. In this way, the use of orthogonal technologies, such as functional testing and immune-profiling, integrated with NGS holds promise to improve outcomes by better matching therapies to individual patients. ${ }^{1}$ In addition, lack of durable efficacy of many categories of therapies has sometimes been attributed to inadequate elimination or targeting of leukemic stem/progenitor cells (reviewed by Rossi et al. ${ }^{2}$ ).

In this issue, Majumder et al. report on the manner by which understanding of innate drug sensitivities in healthy hematopoietic cells advances both the identification of lineage-specific anti-cancer therapies as well as offtarget drug effects in treating acute myeloid leukemia. ${ }^{3}$ Underlying this work is the well-characterized biology of hematopoiesis whereby multipotent stem cells and precursors differentiate through distinct signaling pathways to generate a set of blood cell types with discrete phenotypes and functions. The authors surmise that malignant hematopoietic cells use the same signaling pathways; consequently, they leverage specific pathways from normal cells as a means to identify cancer therapies for their malignant counterparts. Conversely, the authors note that drug responses seen in healthy cells may reveal potential adverse effects.

The authors augment their established cell-based screening platform for identifying anti-leukemia drugs ${ }^{4}$ with high capacity flow cytometry (Figure 1A). This technological development permits the simultaneous evaluation of drug responses from multiple hematopoietic cell populations based on their respective surface antigens. Drug responses are mapped to proteome and cell type specific signaling profiles using mass spectrometry and mass cytometry. In this study, sensitivities to 71 small molecules were simultaneously assessed using multi-parametric flow cytometry and then mapped to proteomic and signaling profiles to characterize the spectrum of drug responses in various hematopoietic cell types. Across healthy cell types for B cells, natural killer (NK) cells, helper $\mathrm{T}$ cells, cytotoxic $\mathrm{T}$ cells and monocytes, the authors identify cell lineage-specific drug responses to define a global view of response profiles. By comparing drug responses between healthy and neoplastic cells, they show that healthy cell responses predict drug responses in corresponding malignant cells. The authors evaluate this screening approach on a large cohort of primary samples obtained from healthy donors and patients with myeloid and lymphoid leukemias, providing evidence that this method identifies new applications for the tested drugs.

A key highlight of this study is the profile observed for the BCL2 inhibitor, venetoclax, which revealed dosedependent sensitivities across the hematopoietic cell types (Figure 1B). At the ends of this spectrum, B cells (CD19+) were the most sensitive whereas monocytes and granulocytes were the least sensitive to venetoclax. Moderate sensitivities were observed on cytotoxic and helper $T$ cells $\left(\mathrm{CD}^{+}{ }^{+} \mathrm{CD} 4\right.$ and $\left.\mathrm{CD}^{+} \mathrm{CD}^{+}\right)$, NK cells $\left(\mathrm{CD}^{-} 6^{+}\right)$, and $\mathrm{NK}-\mathrm{T}$ cells $\left(\mathrm{CD}^{+} \mathrm{CD}^{+} 6^{+}\right)$. Venetoclax had similar cell-specific effects regardless of disease status (healthy vs. malignant) indicating the variable nature of response to venetoclax is lineage specific. In addition, the study found an inverse relationship between venetoclax sensitivity and levels of phosphorylated STAT3. Monocytes and granulocytes have the highest levels of phosphorylated STAT3 and the lowest venetoclax sensitivity, perhaps reflecting the different transcriptional programs defining these two cell types.

Previous work by these authors and others indicated that BCL2 is differentially expressed in subpopulations of AML cells enriched for malignant stem/progenitor cells compared to more differentiated tumor cells ${ }^{5}$ and that venetoclax sensitivity in primary AML cells with a monocytic phenotype is reduced. ${ }^{6}$ These observations led to the hypothesis that clinical features of AML indicative of myeloid differentiation status may correlate with reduced BCL2 dependence in AML patients. Indeed, the venetoclax response profile in this study is consistent with recent findings correlating venetoclax sensitivity with stages of AML disease differentiation as defined by flow cytometry. In the context of venetoclax-based therapies, phenotypically primitive AML is sensitive whereas monocytic AML is more resistant, due to intrinsic properties of monocytic AML cells including loss of BCL2 expression and reliance on MCL1 to mediate oxidative phosphorylation and survival.

Cumulatively, these findings raise the possibility of new definitions for stem/progenitor cells in hematologic malignancies; definitions that would be based on propensity of cell types of any maturation state to persist in the face of a selective pressure. In some ways, these findings may also call into question the long-held notions that targeting 
of more primitive leukemic cell populations will hold the key to durable disease control. It seems possible that most cancer therapeutics utilized to date are simply more active against more mature cell types based on targeting of biological programs that are more prominent in these more mature cells. The identification of drugs that show inversely preferential activity against the more primitive cell states is an example of the enormous benefit that can be derived from this updated platform for flow cytometric drug sensitivity assessment.

Majumder et al. speculate that their profiling will open new opportunities for other disease indications. As examples, they note that dexamethasone and midostaurin targeted NK cells as effectively as B cells, suggesting their potential clinical use in NK-cell malignancies. They further envision incorporating cell lineage specific drug responses into the regimen for preclinical drug development will identify unexpected therapeutic niches for small molecules and enhanced therapeutic precision. Their study provides additional support for the concept of using multiple diagnostic technologies to enhance precision therapy. Indeed, the use of this technology platform to identify

A

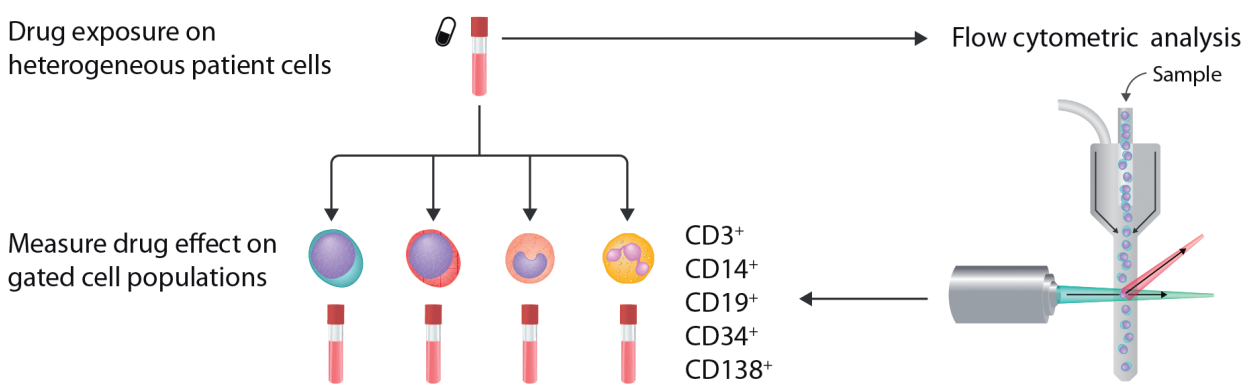

B

Hematopoietic stem cells Differentiated hematopoietic cells

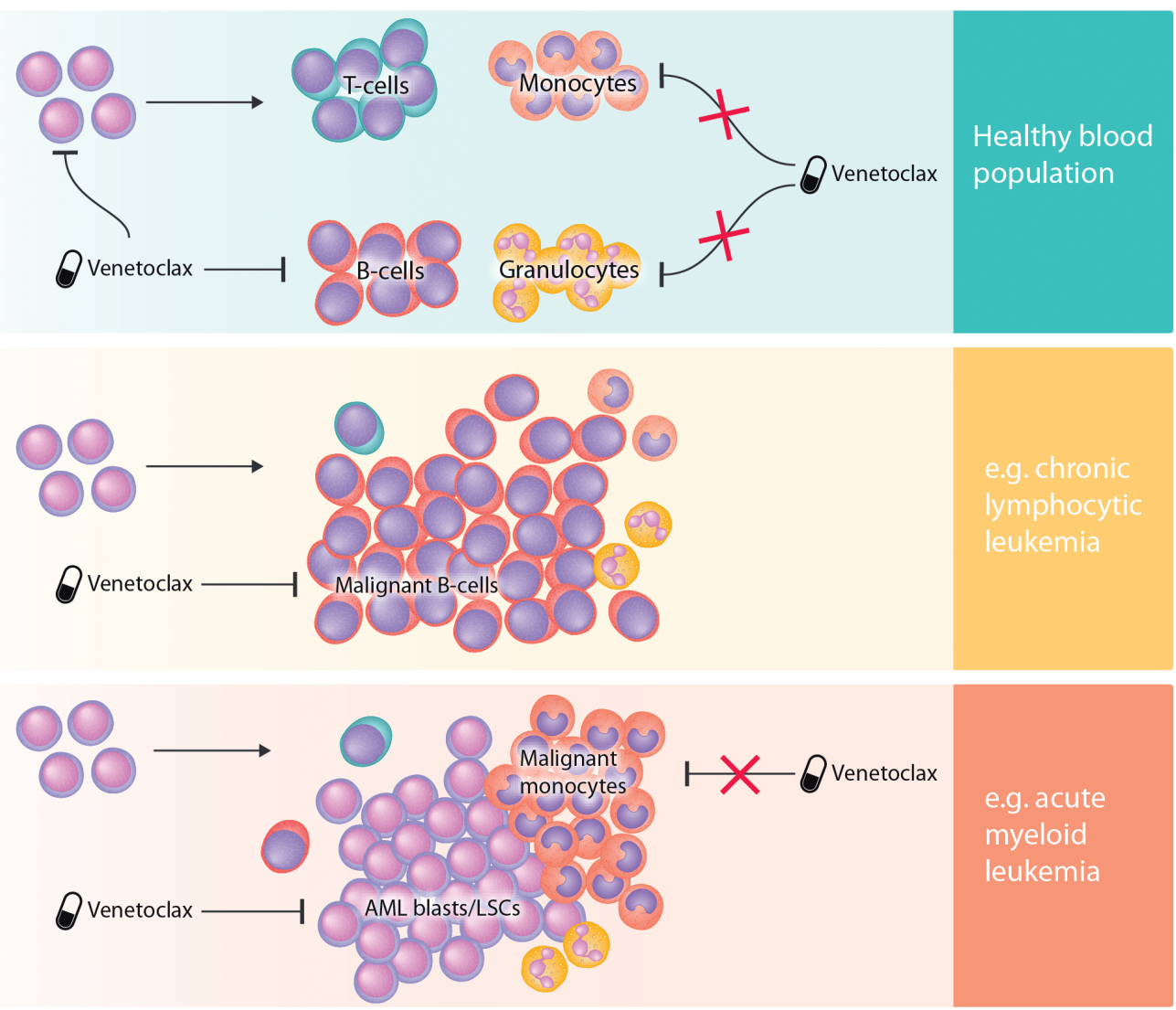

Figure 1. High-throughput flow cytometry functional screening strategy identifies drug effects on discrete cell lineages. (A) Schematic for screening workflow whereby cells from leukemia patient samples are exposed to a library of drugs and then subjected to flow cytometry using antibodies to distinguish discrete cell populations, such as T cells (CD3), B cells (CD19), monocytes (CD14), hematopoietic stem/progenitor cells (CD34), etc. (B) Results from the workflow in (A) have shown that drugs have differential effects on cell lineage states, which are often conserved between malignant and healthy settings. As an example, the BCL2 inhibitor, venetoclax, was shown to have more effect against less differentiated cells as well as mature B cells and less impact on mature monocytes and granulocytes in cells from healthy donors. This observation extended to these same cell states in leukemia patient samples, where venetoclax was more effective in killing mature B cells in chronic lymphocytic leukemia and leukemic progenitor cells in acute myeloid leukemia (AML), but was less effective against malignant monocytes in the same AML samples. 
drug combinations that can simultaneously target the undifferentiated leukemic cell populations as well as the more mature myeloid lineages (especially monocytes) may represent a powerful way to prioritize the most promising drug combinations for pre-clinical study and for clinical development. Clearly, this report demonstrates just the beginning of utility of this exciting drug screening platform.

\section{References}

1. Friedman AA, Letai A, Fisher DE, Flaherty KT. Precision medicine for cancer with next-generation functional diagnostics. Nat Rev Cancer. 2015;15(12):747-756.

2. Rossi DJ, Jamieson CH, Weissman IL. Stems cells and the pathways to aging and cancer. Cell. 2008;132(4):681-696.

3. Majumder MM, Leppa AM, Hellesoy M, et al. Multi-parametric single cell evaluation defines distinct drug responses in healthy hematological cells that are retained in corresponding malignant cell types. Haematologica. 2020;105(6):1527-1538.

4. Pemovska T, Kontro M, Yadav B, et al. Individualized systems medicine strategy to tailor treatments for patients with chemorefractory acute myeloid leukemia. Cancer Discov. 2013;3(12):1416-1429.

5. Lagadinou ED, Sach A, Callahan K, et al. BCL-2 inhibition targets oxidative phosphorylation and selectively eradicates quiescent human leukemia stem cells. Cell Stem Cell. 2013;12(3):329-341.

6. Kuusanmaki H, Leppa AM, Polonen P, et al. Phenotype-based drug screening reveals association between venetoclax response and differentiation stage in acute myeloid leukemia. Haematologica. 2020;105(3):708-720.

7. Pei S, Pollyea DA, Gustafson A, et al. Monocytic Subclones Confer Resistance to Venetoclax-Based Therapy in Patients with Acute Myeloid Leukemia. Cancer Discov. 2020;10(4):536-551.

\title{
To target the untargetable: elucidation of synergy of APR-246 and azacitidine in TP53 mutant myelodysplastic syndromes and acute myeloid leukemia
}

\author{
David A. Sallman
}

\author{
Department of Malignant Hematology, H. Lee Moffitt Cancer Center, Tampa, FL, USA \\ E-mail: DAVID A. SALLMAN - david.sallman@moffitt.org
}

doi:10.3324/haematol.2020.249060

M utations of the tumor suppressor gene TP53 represent a common mutation in myeloid malignancies, occurring in 10-20\% of patients with de novo myelodysplastic syndromes (MDS) and acute myeloid leukemia (AML) with profound negative impact on outcomes and a median overall survival (OS) of 6-12 months. ${ }^{1 .}$ ${ }^{3}$ Critically, the clonal burden of TP53, that is the variant allele frequency (VAF) and/or allelic state of TP53, is intimately tied with the clinical trajectory of these patients and is a robust, independent predictor of survival. ${ }^{47}$ Given the poor OS and lack of therapeutic options for TP53 mutant MDS/AML patients, a number of novel agents are being investigated in this patient group. ${ }^{8}$ Of these, APR246 has evoked considerable excitement based on its robust clinical efficacy in combination with azacitidine in TP53 mutant MDS/AML patients. ${ }^{9,10}$ In this issue of Haematologica, ${ }^{11}$ Maslah et al. describe compelling preclinical synergy of APR-246 in combination with azacitidine in TP53 mutated MDS and AML and, more importantly, identify a novel molecular mechanism underlying the observed synergy.

Recent elegant work has definitively identified that TP53 missense mutations in myeloid malignancies result in a dominant-negative effect without evidence of neomorphic gain-of-function activities, ultimately leading to a selection advantage when exposed to DNA damage. ${ }^{12}$ Thus, restoring wild-type function in TP53 mutant clones would be of profound beneficial impact. APR-246, a methylated PRIMA-1 analog, is a novel, first-in-class, small molecule that selectively induces apoptosis in TP53 mutant cancer cells. Mechanistically, APR-246 is spontaneously converted into the active species methylene quinuclidinone (MQ), which is able to covalently bind to cysteine residues in mutant p53 thereby producing thermo- dynamic stabilization of the protein and shifting equilibrium toward a functional conformation. ${ }^{13,14}$ APR-246 monotherapy was originally investigated in a phase I trial including AML patients with clinical activity and correlative data identifying activation of p53-dependent pathways. ${ }^{15,16}$

Maslah et al. identified in TP53 mutant cell lines, in vivo models, and primary patient samples that the combination of APR-246 and azacitidine results in a synergistic pro-apoptotic effect as well as a dramatic reduction in cell proliferation via cell cycle arrest (Figure 1). As the majority of TP53 mutations are missense and located in the DNA binding domain, synergy experiments were performed with the SKM1 cell line, which harbors a homozygous hotspot mutation of TP53 (p. R248Q), and thus is an appropriate representation of clinical disease. ${ }^{17}$ Combination therapy of APR-246 and azacitidine resulted in a doubling of apoptotic cells versus azacitidine alone as well as $83 \%$ of cells undergoing cell cycle arrest in G0/G1. This synergistic effect was confirmed in a xenotransplantation model where combination therapy resulted in a pronounced inhibition of disease progression which occurred early and was durable. Subsequently, the authors interrogated differential gene expression profiles of SKM1 cells treated with either drug alone versus the combination of APR-246 and azacitidine. As expected, Gene Set Enrichment Analysis (GSEA) and DAVID analyses of APR246 treated cells showed robust induction of p53-target genes including CDKN1A, CASP1, BAX and FAS, which was confirmed by reverse transcription real-time quantitative polymerase chain reaction (RT-qPCR), resulting in activation of an early apoptotic program. Furthermore, GSEA analysis of "synergistic only" genes (i.e. genes differentially expressed only with combination treatment) 